\title{
Localised upper airway obstruction in a patient with acquired immunodeficiency syndrome
}

\author{
Christine L D’Arsigny, Sally Ford, Denis E O'Donnell
}

\begin{abstract}
Summary
We describe a case of rapidly progressive upper airway obstruction due to tracheal Pseudomonas abscesses in a patient with acquired immunodeficiency syndrome. The case highlights the aggressive nature of Pseudomonas infections and the difficulty of eradicating this organism in patients infected with the human immunodeficiency virus.
\end{abstract}

Keywords: airway obstruction; Pseudomonas infection; tracheal abscess; acquired immunodeficiency syndrome

A 40-year-old homosexual man, with a 10 -year history of human immunodeficiency virus (HIV) infection, was admitted to hospital with 2 weeks of worsening shortness of breath, fever and cough. Three months previously, he had been admitted with Pseudomonas aeruginosa bacteraemia. One month later, he was readmitted with sinusitis and a new left upper lobe infiltrate. Blood and sputum cultures were negative and he improved on empiric antipseudomonas antibiotics. He was admitted on 1 January 1996 with a febrile neutropenia (leucocytes $=0.4 \times 10^{9} / 1$, normal range 4.0-11.0) with a neutrophil nadir of 0.06 (normal range $2.0-7.5 \times 10^{9} / 1$ ) and cough. This was on a background of chronic mild neutropenia over the previous year and was presumed to be aggravated by therapy with azido-thymidine (AZT) and rifabutin. However, discontinuation of these drugs resulted in only partial recovery of leucocytes to $0.9 \times 10^{9} / 1$. Blood cultures again were negative and chest radiographs remained unchanged from the previous admission (figure 1). He showed clinical improvement after 2 weeks of antibiotics and empiric antifungal therapy (fluconazole, 800 mg once daily). He was admitted again one month later with fever and cough. Blood cultures were again negative and the chest radiograph remained unchanged from the previous admission. He showed clinical improvement after 2 weeks of antibiotics and was discharged. In the 2 weeks prior to his most recent admission, he developed progressive dyspnoea, productive cough, fever, and chills. The patient had no history of respiratory tract infections with Pneumocystis carinii or tuberculosis.

Physical examination revealed a thin, lethargic-looking man in moderate respiratory distress. His respiratory rate was 28 breaths/ min, blood pressure $180 / 70 \mathrm{mmHg}$, heart rate 107 beats $/ \mathrm{min}$, and his temperature was $39^{\circ} \mathrm{C}$. There were findings suggestive of oral candidiasis. Pulmonary auscultation revealed diminished breath sounds, but no wheezing or stridor. Laboratory values revealed a white count of $6.6 \times 10^{9} / 1$ with neutrophils of $3.1 \times$ $10^{9} / 1$; his CD4 count was 4 . His haemoglobin was $10.8 \mathrm{~g} / \mathrm{dl}$, platelets were $321 \times 10^{9} / 1$, and electrolytes and renal function were normal. The lactate dehydrogenase $(\mathrm{LDH})$ was elevated at $1208 \mathrm{IU} / 1\left(\mathrm{LDH} / \mathrm{PaO}_{2}=16.7\right)$, with an alkaline phosphatase of 652 IU/1. Transami-

\author{
Division of \\ Respiratory \& Critical \\ Care Medicine, \\ Department of \\ Medicine, Queen's \\ University, Richardson \\ House, 102 Stuart \\ Street, Kingston \\ General Hospital, \\ Kingston, Ontario, \\ Canada K7L 2V7 \\ C L D'Arsigny \\ $S$ Ford \\ D E O'Donnell
}

Correspondence to

Dr Denis E O’Donnel

Accepted 23 April 1998
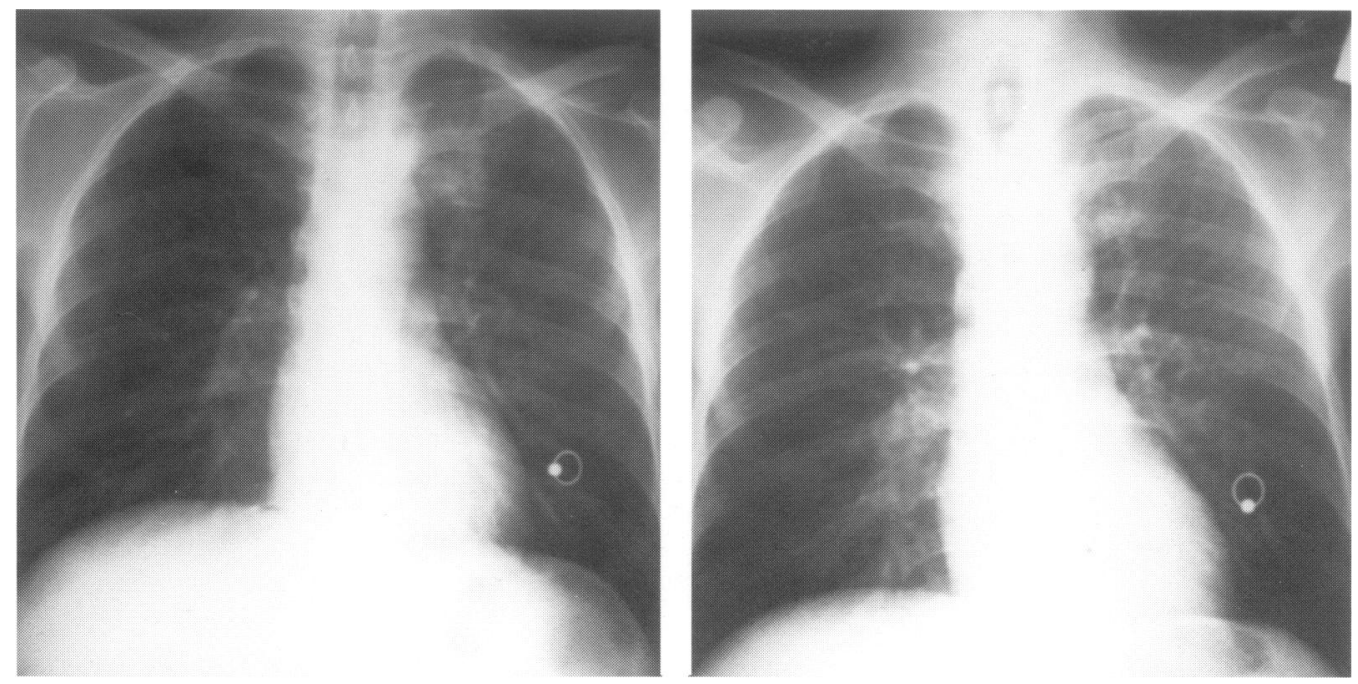

Figure 1 Chest radiograph from 1 January 1996 on the left and from 30 January on the right. The second film shows narrowing of the tracheal air column and no progression of the left upper lobe lesion 


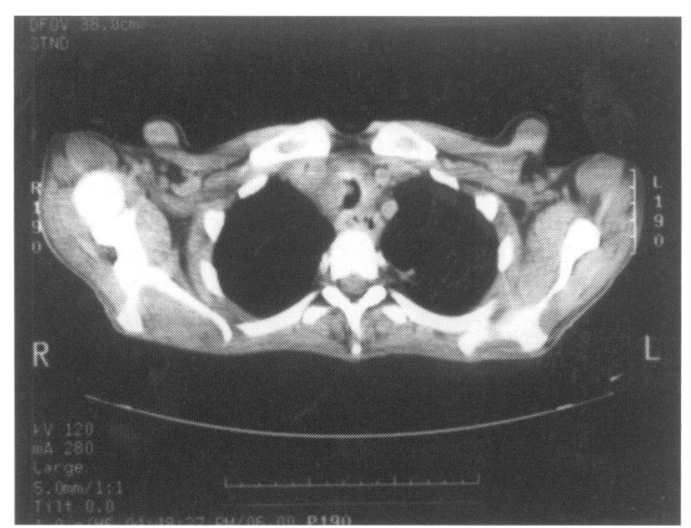

Figure 2 CT scan of the upper airway. Obstruction of the mid-trachea is evident with $>50 \%$ reduction in its internal diameter. A right extrinsic tracheal mass is seen protruding into the tracheal lumen

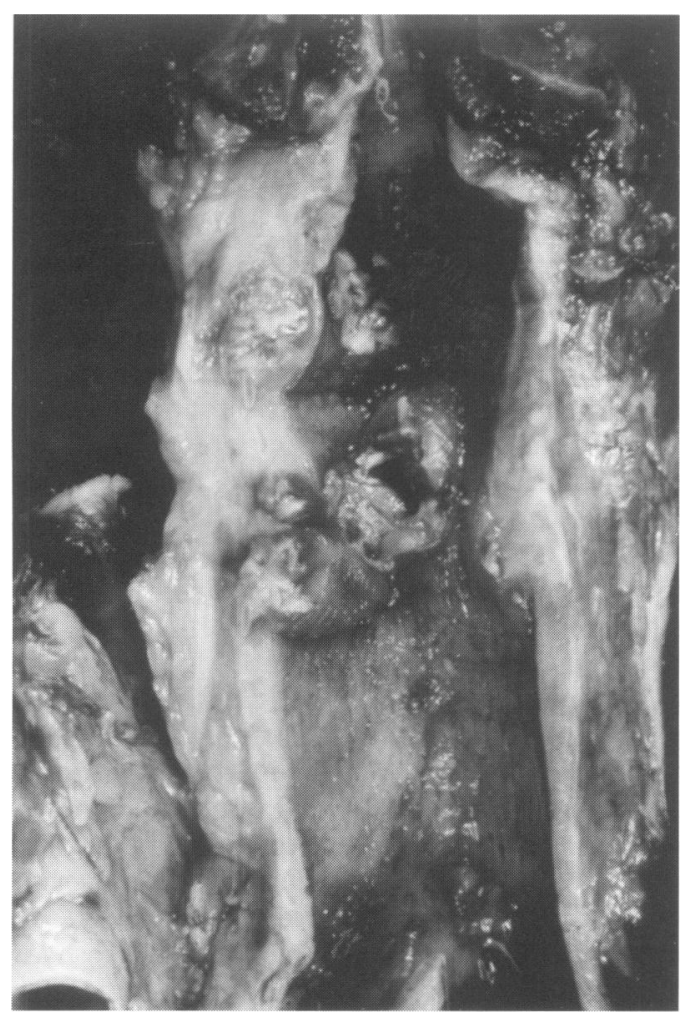

Figure 3 Maximal flow-volume loop in the patient (solid line) compared with age-matched predicted loop. Note severe reduction in maximal expiratory and inspiratory flows: expiratory flow rates are disproportionately reduced

nases and bilirubin were normal. Arterial blood gases on room air yielded a pH of $7.42, \mathrm{PaO}_{2}$ of $9.6 \mathrm{kPa}, \mathrm{PaCO}_{2}$ of $4.9 \mathrm{kPa}$, and bicarbonate of $24 \mathrm{mmol} / \mathrm{l}$. A chest radiograph identified an unexpected, new narrowing of the tracheal air column compared to a film one month earlier (figure 1, right). The ill-defined left upper lobe infiltrate, which was first noted six weeks earlier, was still present, but moderately improved.

Helical computed tomography (CT) of the trachea confirmed the obstruction in the midtrachea with $>50 \%$ reduction in the internal lumen (figure 2). There was also a large, soft tissue density within the tracheal wall with extension into the tracheal lumen. A CT scan



Figure 4 Pathologic specimen of the trachea and surrounding tissues. Multiple ulcerated lesions are seen with thickening of the tracheal wall. The internal diameter is reduced by $>75 \%$

of the chest identified a poorly demarcated infiltrate with a cystic component in the left upper lobe, and moderate bilateral air space disease which was not appreciated on the chest radiograph the previous day.

A maximal flow-volume loop was performed to evaluate the functional significance of anatomical airway narrowing (figure 3 ). This showed markedly diminished maximal expiratory flows: peak expiratory flow rate $=1.2 \mathrm{l} / \mathrm{s}$ ( $14 \%$ predicted); maximal mid-expiratory flow rate $=0.35 \mathrm{l} / \mathrm{s}(9.4 \%$ predicted); maximal flow at $75 \%$ of vital capacity (VC) was $0.52 \mathrm{l} / \mathrm{s}$ $(6.8 \%$ predicted) and at $25 \%$ of the VC was $0.26 \mathrm{l} / \mathrm{s}$ (9.9\% predicted); peak inspiratory flow rate $=1.7 \mathrm{l} / \mathrm{s}$ ( $28 \%$ predicted). The disproportionate reduction in expiratory flows relative to inspiratory flows over the mid-volume range suggest a component of variable intrathoracic upper airway obstruction, as well as fixed upper airway obstruction pattern (figure 3).

Bronchoscopy revealed severe narrowing of the mid-trachea, spanning 3-5 cm in length with a large ulcerated lesion along the anterior tracheal wall. There were copious amounts of muco-purulent secretions. Gram staining revealed large quantities of Gram-negative organisms, and staining for acid-fast bacilli and Pneumocystis carinii were negative. While awaiting final bronchial culture results, the patient was started on broad-spectrum, antipseudomonal antibiotics.

The patient declined intubation or resuscitation in the event of further clinical deterioration. He was, therefore, treated with chest physiotherapy, suctioning and close assessment of his respiratory status, along with the broadspectrum antibiotics. The patient died acutely 
during sleep from a respiratory arrest, 56 hours after admission.

An autopsy was obtained and showed $75 \%$ stenosis of the tracheal lumen (figure 4). There were multiple tracheal ulcerations within the lumen with areas of ulceration as well as abscesses within the tracheal wall with diffuse destruction of cartilage, suggesting the potential for dynamic instability of the trachea. There was also peritracheal fibrosis suggesting relative chronicity of the lesion. The patient's lung parenchyma had diffuse haemorrhage and micro-abscesses, along with a cavity in the left upper lobe. Tracheal brushings and sputum cultures showed heavy growths of Pseudomonas aeruginosa, sensitive to the antibiotics that he was receiving. Fungal cultures from tracheal brushings and lung secretions were negative, and no other pathogens were isolated.

\section{Discussion}

The presence of localised upper airway obstruction in HIV is rare and sparsely described in the literature. Different aetiologic possibilities include bacterial infections ${ }^{1}$ and infections with cytomegalovirus, ${ }^{2}$ herpes simplex virus, and fungal organisms. ${ }^{3}$ Tracheal malignancy, including primary and metastatic carcinoma, lymphoma and Kaposi's sarcoma ${ }^{4}$ have also been reported. Although an inflammatory process was suspected in our patient due to the rapid progression of the airway narrowing, the possibility of localised Pseudomonas abscesses was not specifically entertained and, to our knowledge, has not previously been described in HIV patients. There is one report of mixed bacterial tracheitis from Staphylococcus aureus and Pseudomonas aeruginosa by Valor et al, ${ }^{1}$ but their patient did not have a discrete abscess.

The mechanism of formation for the tracheal abscesses in our patient was not ascertained. However, given this patient's history of previous Pseudomonas bacteraemia, we suspect that his chronic sinusitis and lung infections were also caused by the same organism. The autopsy findings were suggestive of a chronic inflammatory process in the trachea (ie, peritracheal fibrosis and cartilage erosion), with lack of a demonstrable communication from the lung

1 Valor RR, Polnitsky CA, Tanis DJ, Sherter CB. Bacterial tracheitis with upper airway obstruction in a patient with the acquired immunodeficiency syndrome. Am Rev Resp Di 1992;146:1598-9.

2 Imoto EM, Stein RM, Shellito JE, Curtis JL. Central airway obstruction due to cytomegalovirus-induced necrotizing tracheitis in a patient with AIDS. Am Rev Resp Dis 1990;142:884-6.

3 Patete ML, Mellon D. Extrinsic tracheal compression-a newly described complication of acquired immune deficiency syndrome (AIDS). Ear Nose Throat f 1995;74:189-92.

\section{Learning points}

- critical airway narrowing can occur in the absence of physical signs (ie, stridor)

- fulminant bilateral pseudomonal pneumonia can occur in the HIV-infected host in the presence of only minor radiographic abnormality

- consider pseudomonal abscess formation in patients with HIV presenting with localised upper airway obstruction

- chronic pseudomonal infection in patients with HIV are difficult to eradicate and carry a high mortality

abscesses to the trachea. Tracheal infection may, therefore, have been related to chronic pseudomonal sinusitis. Although multiple pathogens may co-exist in the lungs in end-stage HIV infection, only Pseudomonas was cultured from the trachea and lung secretions in this patient. Other potential pathogens, including fungi, were not identified.

Patients with HIV have abnormal host defence mechanisms which may predispose them to Pseudomonas infections. ${ }^{5}$ Chronic neutropenia, aggravated by antiviral agents, may have additionally predisposed this patient to persistent Pseudomonas infection. Despite appropriate antibiotic therapy, a third of HIV patients have recurrent infections. ${ }^{6}$ Any site of recurrence is possible and mortality then escalates to $80 \%$.

Although the cause of this patient's death was multifactorial, critical airway narrowing probably contributed to his demise. We speculate that the tracheal occlusion was due to both dynamic instability of the trachea from cartilage erosion and structural narrowing from extraluminal compression and tracheal abscesses. That the airway narrowing was critically reduced is verified by the severe obstructive pattern on the maximal flow-volume loop. This patient also had fulminant bilateral Pseudomonas pneumonia and an inability to clear his secretions which, in combination with the critical airway narrowing, probably culminated in his demise.

4 Beitler AJ, Ptaszynski K, Karpel JP. Upper airway obstruction in a woman with AIDS-related laryngeal Kaposi's sarcoma. Chest 1996;109:836-7.

5 Kielhofner M, Atmar RL, Hamill RJ, Musher DM Life-threatening Pseudomonas aeruginosa infections in patients with human immunodeficiency virus infection. Clin Infect Dis 1992;14:403-11.

6 Mendelson MH, Gurtman A, Szabo S, et al. Pseudomonas aeruginosa bacteremia in patients with AIDS. Clin Infect Dis 1994;18:886-95. 